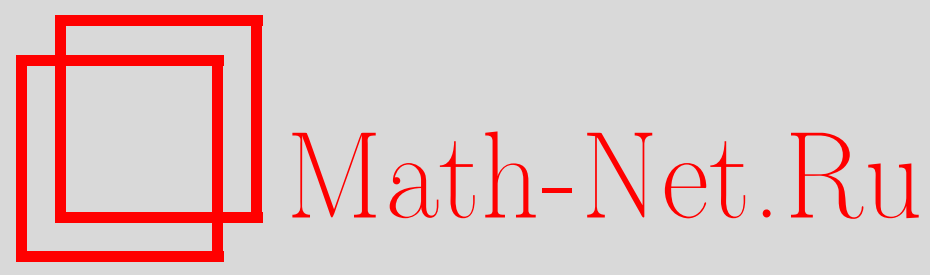

Н. А. Ильясов, О порядке убывания равномерных модулей гладкости на классах функций $E_{p, m}[\epsilon]$, Матем. заметки, 2005, том 78, выпуск 4, 519-536

DOI: https://doi.org/10.4213/mzm2611

Использование Общероссийского математического портала Math-Net.Ru подразумевает, что вы прочитали и согласны с пользовательским соглашением http://www. mathnet.ru/rus/agreement

Параметры загрузки:

IP : 54.224.135.184

26 апреля 2023 г., 12:50:31

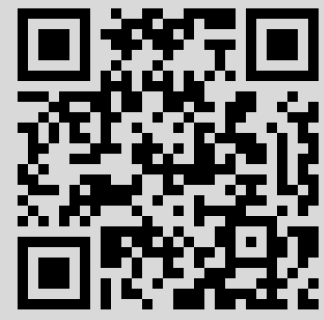




\section{О ПОРЯДКЕ УБЫВАНИЯ РАВНОМЕРНЫХ МОДУЛЕЙ ГЛАДКОСТИ НА КЛАССАХ ФУНКЦИЙ $E_{p, m}[\varepsilon]$}

\section{Н. А. Ильясов}

В заметке приводится решение задачи о точном порядке убывания равномерных модулей гладкости на классах $2 \pi$-периодических функций многих переменных с заданной мажорантой последовательности полных наилучших в метрике $L_{p}$ приближений, $1 \leqslant p<\infty$.

Библиография: 16 названий.

Пусть $\mathbb{R}^{m}-m$-мерное евклидово пространство точек $x=\left(x_{1}, \ldots, x_{m}\right)$ с нормой $|x|=\left(x_{1}^{2}+\cdots+x_{m}^{2}\right)^{1 / 2}, m \in \mathbb{N}$,

$$
\mathbb{T}^{m}=[-\pi, \pi]^{m}=\left\{x \in \mathbb{R}^{m} ;-\pi \leqslant x_{i} \leqslant \pi, i=\overline{1, m}\right\},
$$

$L_{p}\left(\mathbb{T}^{m}\right), 1 \leqslant p<\infty,-$ пространство всех измеримых $2 \pi$-периодических по каждой переменной $x_{i}, i=\overline{1, m}$, функций $f(x)=f\left(x_{1}, \ldots, x_{m}\right)$, для которых

$$
\|f\|_{L_{p}\left(\mathbb{T}^{m}\right)} \equiv\|f\|_{p, m}=\left\{\pi^{-m} \int_{\mathbb{T}^{m}}|f(x)|^{p} d x\right\}^{1 / p}<\infty,
$$

$L_{\infty}\left(\mathbb{T}^{m}\right) \equiv C\left(\mathbb{T}^{m}\right)$ - соответствующее пространство непрерывных функций,

$$
\|f\|_{C\left(\mathbb{T}^{m}\right)}=\|f\|_{\infty, m}=\max \left\{|f(x)|: x \in \mathbb{T}^{m}\right\},
$$

$E_{n_{1}, \ldots, n_{m}}(f)_{p, m}$ - полное наилучшее в метрике $L_{p}\left(\mathbb{T}^{m}\right)$ приближение функции $f$ тригонометрическими полиномами порядка $\leqslant n_{i} \in \mathbb{Z}_{+}$соответственно по переменной $x_{i}$, $i=\overline{1, m} ; \omega_{k}(f ; \delta)_{p, m}$ - полньй модуль гладкости $k$-го порядка функции $f \in L_{p}\left(\mathbb{T}^{m}\right)$, $k \in \mathbb{N}$ :

$$
\omega_{k}(f ; \delta)_{p, m}=\sup \left\{\left\|\Delta_{h}^{k} f(\cdot)\right\|_{p, m}: h \in \mathbb{R}^{m},|h| \leqslant \delta\right\}, \quad \delta>0,
$$

где

$$
\Delta_{h}^{k} f(x)=\sum_{\nu=0}^{k}(-1)^{k-\nu}\left(\begin{array}{l}
k \\
\nu
\end{array}\right) f(x+\nu h), \quad x \in \mathbb{R}^{m},
$$

$\mathscr{M}_{0}-$ класс всех числовых последовательностей $\varepsilon=\left\{\varepsilon_{n}\right\}_{n=1}^{\infty}$ таких, что $0<\varepsilon_{n} \downarrow 0$ при $n \uparrow \infty$.

Обозначим (при $\left.1 \leqslant p<\infty, \varepsilon \in \mathscr{M}_{0}\right)$

$$
E_{p, m}[\varepsilon]=\left\{f \in L_{p}\left(\mathbb{T}^{m}\right) ; E_{n-1, \ldots, n-1}(f)_{p, m} \leqslant \varepsilon_{n}, n \in \mathbb{N}\right\} .
$$

В настоящей заметке приводится решение задачи о точном порядке убывания равномерных модулей гладкости $k$-го порядка функций из классов $E_{p, m}[\varepsilon]$, где $k \in \mathbb{N}$, $1 \leqslant p<\infty$. Отметим, что в случае $m=1$ решение этой задачи приведено автором в [1]. 
Teорема. Пусть $k \in \mathbb{N}, 1 \leqslant p<\infty, m \in \mathbb{N}, \varepsilon \in \mathscr{M}_{0}, d=\pi m^{1 / 2}$ u сходится ряд

$$
\sum_{n=1}^{\infty} n^{m / p-1} \varepsilon_{n}<\infty .
$$

Тогда

$$
\begin{aligned}
& \sup \left\{\omega_{k}\left(\psi ; \frac{d}{n}\right)_{\infty, m} ; f \in E_{p, m}[\varepsilon]\right\} \\
& \underset{\left(k, \widehat{m}_{, p}\right)}{\sum_{\nu=n+1}^{\infty}} \nu^{m / p-1} \varepsilon_{\nu}+n^{-k} \sum_{\nu=1}^{n} \nu^{k+m / p-1} \varepsilon_{\nu}, \quad n \in \mathbb{N},
\end{aligned}
$$

где $\psi$ обозначает соответствующую функиию из $C\left(\mathbb{T}^{m}\right)$, әквивалентную $f \in$ $E_{p, m}[\varepsilon]$, что обеспечивается условием (1). (Здесь $\alpha_{n} \underset{(k, m, p)}{\asymp} \beta_{n}$ обозначает существование таких постоянных $0<C_{1} \leqslant C_{2}$, зависящих лишь от указанных параметров $k, m$ и $p$, что $C_{1} \cdot \beta_{n} \leqslant \alpha_{n} \leqslant C_{2} \cdot \beta_{n}$.)

Доказательство теоремы основывается на следуюших леммах.

Лемма 1. Пусть $1 \leqslant p<\infty, m \in \mathbb{N}, f \in L_{p}\left(\mathbb{T}^{m}\right), k \in \mathbb{N} u$ сходится ряд

$$
\sum_{n=1}^{\infty} n^{m / p-1} E_{n-1, \ldots, n-1}(f)_{p, m}<\infty .
$$

Тогда $f$ әквивалентна (в смысле т-мерной меры Лебега) некоторой функции $\psi \in C\left(\mathbb{T}^{m}\right)$ и справедлива оченка

$$
\begin{aligned}
\omega_{k}\left(\psi ; \frac{d}{n}\right)_{\infty, m} \leqslant & C_{1}(k, p, m)\left\{\sum_{\nu=n+1}^{\infty} \nu^{m / p-1} E_{\nu-1, \ldots, \nu-1}(f)_{p, m}\right. \\
& \left.+n^{-k} \sum_{\nu=1}^{n} \nu^{k+m / p-1} E_{\nu-1, \ldots, \nu-1}(f)_{p, m}\right\}, \quad n \in \mathbb{N} .
\end{aligned}
$$

ДокАЗАТЕЛЬСтво. Утверждение леммы 1 в случае $m=1$ приведено в $[2$, с. 57$]$ в качестве следствия неравенства (1.8) из теоремы 2 и неравенства (1.7) из следствия 1 (см. $[2$, с. 56$])$. Другое доказательство леммы 1 в случае $m=1$ приведено также в $[3$, c. 378-379]. Ниже приводится доказательство леммы 1 в случае $m>1$.

$\mathrm{B}[4$, c. 141$]$ отмечен $m$-мерньй аналог неравенства Конюшкова-Стечкина (при $m=1$ см. $[2$, неравенство $(1.8)$, с. 56$])$, из доказательства (проводимого точно так же, как и в одномерном случае) которого, в частности, следует, что если для функции $f \in L_{p}\left(\mathbb{T}^{m}\right)$ сходится ряд (3), то $f$ эквивалентна некоторой функции $\psi \in C\left(\mathbb{T}^{m}\right)$ и справедлива оценка (при $n \in \mathbb{Z}_{+}$)

$$
\begin{aligned}
& E_{n, \ldots, n}(\psi)_{\infty, m} \leqslant\left\|\psi(\cdot)-T_{n ; p}^{(m)}(f ; \cdot)\right\|_{\infty, m} \\
& \quad \leqslant C_{2}(p, m)\left\{(n+1)^{m / p} E_{n, \ldots, n}(f)_{p, m}+\sum_{\nu=n+1}^{\infty} \nu^{m / p-1} E_{\nu, \ldots, \nu}(f)_{p, m}\right\},
\end{aligned}
$$


где $T_{n ; p}^{(m)}(f ; x) \equiv T_{n ; \ldots, n ; p}(f ; x)$ - тригонометрический полином полного наилучшего в $L_{p}\left(\mathbb{T}^{m}\right)$ приближения функции $f$ порядка $n \in \mathbb{Z}_{+}$по переменной $x_{i}, i=\overline{1, m}$ :

$$
\left\|f(\cdot)-T_{n ; p}^{(m)}(f ; \cdot)\right\|_{p, m}=E_{n, \ldots, n}(f)_{p, m} .
$$

Далее, в силу известных свойств модулей гладкости (см., например, [5, с. 174-178]) при $\alpha=\left(\alpha_{1}, \ldots, \alpha_{m}\right), \alpha_{i} \in \mathbb{Z}_{+}, i=\overline{1, m},|\alpha|=\alpha_{1}+\cdots+\alpha_{m}, \alpha !=\alpha_{1} ! \cdots \alpha_{m}$ ! имеем

$$
\begin{aligned}
\omega_{k}(\psi ; \delta)_{\infty, m} & \leqslant \omega_{k}\left(\psi-T_{n ; p}^{(m)}(f) ; \delta\right)_{\infty, m}+\omega_{k}\left(T_{n ; p}^{(m)}(f) ; \delta\right)_{\infty, m} \\
& \leqslant 2^{k}\left\|\psi(\cdot)-T_{n ; p}^{(m)}(f ; \cdot)\right\|_{\infty, m}+\delta^{k} \sum_{|\alpha|=k} \frac{k !}{\alpha !}\left\|D^{\alpha} T_{n ; p}^{(m)}(f ; \cdot)\right\|_{\infty, m} \\
& \leqslant 2^{k}\left\|\psi(\cdot)-T_{n ; p}^{(m)}(f ; \cdot)\right\|_{\infty, m}+m^{k} \delta^{k} \max _{|\alpha|=k}\left\|D^{\alpha} T_{n ; p}^{(m)}(f ; \cdot)\right\|_{\infty, m} .
\end{aligned}
$$

$\left(\right.$ Здесь $D^{\alpha} g(x)=\partial^{|\alpha|} g\left(x_{1}, \ldots, x_{m}\right) / \partial x_{1}^{\alpha_{1}} \ldots \partial x_{m}^{\alpha_{m}}$.)

Оценим сверху $\left\|D^{\alpha} T_{n ; p}^{(m)}(f ; \cdot)\right\|_{\infty, m}$ при $|\alpha|=k$ через $\left\{E_{\nu-1, \ldots, \nu-1}(f)_{p, m}\right\}_{\nu=1}^{n}$, следуя схеме рассуждений Стечкина [6, с. 233-234] (см. также [3, с. 345-346]). Зафиксируем $n \in \mathbb{N}$ и определим $l \in \mathbb{N}$ из условий $2^{l-1} \leqslant n<2^{l}$. В силу очевидного представления

$$
\begin{aligned}
T_{n ; p}^{(m)}(f ; x)= & T_{0 ; p}^{(m)}(f ; x)+\sum_{\nu=1}^{l-1}\left\{T_{2^{\nu}-1 ; p}^{(m)}(f ; x)-T_{2^{\nu-1}-1 ; p}^{(m)}(f ; x)\right\} \\
& +T_{n ; p}^{(m)}(f ; x)-T_{2^{l-1}-1 ; p}^{(m)}(f ; x),
\end{aligned}
$$

имеем

$$
\begin{aligned}
D^{\alpha} T_{n ; p}^{(m)}(f ; x)= & \sum_{\nu=1}^{l-1}\left\{D^{\alpha} T_{2^{\nu}-1 ; p}^{(m)}(f ; x)-D^{\alpha} T_{2^{\nu-1}-1 ; p}^{(m)}(f ; x)\right\} \\
& +D^{\alpha} T_{n ; p}^{(m)}(f ; x)-D^{\alpha} T_{2^{l-1}-1 ; p}^{(m)}(f ; x)
\end{aligned}
$$

откуда в силу $m$-мерных аналогов неравенства Бернштейна-Рисса-Зигмунда об оценке $L_{p}$-норм производных тригонометрических полиномов и неравенства разных метрик Джексона-Никольского для тригонометрических полиномов (см., например, [3, неравенство (30) на с. 232 , неравенство (7) на с. 243 , неравенство (18) на с. 246], [5, с. 150, 159]) имеем (при $2^{l-1} \leqslant n<2^{l}$, т.е. $2^{l-1}-1<n \leqslant 2^{l}-1$ )

$$
\begin{aligned}
& \left\|D^{\alpha} T_{n ; p}^{(m)}(f ; \cdot)\right\|_{\infty, m} \\
& \leqslant \sum_{\nu=1}^{l-1}\left\|D^{\alpha}\left\{T_{2^{\nu}-1 ; p}^{(m)}(f ; \cdot)-T_{2^{\nu-1}-1 ; p}^{(m)}(f ; \cdot)\right\}\right\|_{\infty, m} \\
& \quad+\left\|D^{\alpha}\left\{T_{n ; p}^{(m)}(f ; \cdot)-T_{2^{l-1}-1 ; p}^{(m)}(f ; \cdot)\right\}\right\|_{\infty, m} \\
& \quad 2^{m} \sum_{\nu=1}^{l-1}\left(2^{\nu}-1\right)^{|\alpha|+m / p}\left\|T_{2^{\nu}-1 ; p}^{(m)}(f ; \cdot)-T_{2^{\nu-1}-1 ; p}^{(m)}(f ; \cdot)\right\|_{p, m} \\
& \quad+2^{m} n^{|\alpha|+m / p}\left\|T_{n ; p}^{(m)}(f ; \cdot)-T_{2^{l-1}-1 ; p}^{(m)}(f ; \cdot)\right\|_{p, m}
\end{aligned}
$$




$$
\begin{aligned}
\leqslant & 2^{m} \sum_{\nu=1}^{l-1}\left(2^{\nu}-1\right)^{|\alpha|+m / p}\left\{E_{2^{\nu}-1, \ldots, 2^{\nu}-1}(f)_{p, m}+E_{2^{\nu-1}-1, \ldots, 2^{\nu-1}-1}(f)_{p, m}\right\} \\
& +2^{m} n^{|\alpha|+m / p}\left\{E_{n, \ldots, n}(f)_{p, m}+E_{2^{l-1}-1, \ldots, 2^{l-1}-1}(f)_{p, m}\right\} \\
\leqslant & 2^{m+1} \sum_{\nu=1}^{l-1}\left(2^{\nu}-1\right)^{k+m / p} E_{2^{\nu-1}-1, \ldots, 2^{\nu-1}-1}(f)_{p, m} \\
& +2^{m+1}\left(2^{l}-1\right)^{k+m / p} E_{2^{l-1}-1, \ldots, 2^{l-1}-1}(f)_{p, m} \\
= & 2^{m+1} \sum_{\nu=1}^{l}\left(2^{\nu}-1\right)^{k+m / p} E_{2^{\nu-1}-1, \ldots, 2^{\nu-1}-1}(f)_{p, m} \\
= & 2^{m+1}\left\{E_{0, \ldots, 0}(f)_{p, m}+\sum_{\nu=2}^{l}\left(2^{\nu}-1\right)^{k+m / p} E_{2^{\nu-1}-1, \ldots, 2^{\nu-1}-1}(f)_{p, m}\right\} \\
\leqslant & 2^{m+1}\left\{E_{0, \ldots, 0}(f)_{p, m}+C_{3}(k, p, m) \sum_{\nu=2}^{l} \sum_{\mu=2^{\nu-2}}^{2^{\nu-1}-1} \mu^{k+m / p-1} E_{\mu, \ldots, \mu}(f)_{p, m}\right\} \\
\leqslant & 2^{m+1}\left\{E_{0, \ldots, 0}(f)_{p, m}+C_{3}(k, p, m) \sum_{\mu=1}^{l-1} \mu^{k+m / p-1} E_{\mu, \ldots, \mu}(f)_{p, m}\right\} \\
& \quad k, p, m) \sum_{\mu=1}^{n} \mu^{k+m / p-1} E_{\mu-1, \ldots, \mu-1}(f)_{p, m},
\end{aligned}
$$

где $C_{3}(k, p, m)=\left(2^{k+m / p}-1\right)^{-1}(k+m / p) 6^{k+m / p}, C_{4}(k, p, m)=2^{m+1} C_{3}(k, p, m)$, и, следовательно,

$$
\max _{|\alpha|=k}\left\|D^{\alpha} T_{n ; p}^{(m)}(f ; \cdot)\right\|_{\infty, m} \leqslant C_{4}(k, p, m) \sum_{\nu=1}^{n} \nu^{k+m / p-1} E_{\nu-1, \ldots, \nu-1}(f)_{p, m} .
$$

Учитьвая теперь оценки (5) и (7) в неравенстве (6) (положим $\delta=d / n)$, получим $\begin{aligned} \omega_{k}\left(\psi ; \frac{d}{n}\right)_{\infty, m} \leqslant & 2^{k}\left\|\psi(\cdot)-T_{n ; p}^{(m)}(f ; \cdot)\right\|_{\infty, m}+m^{k} d^{k} n^{-k} \max _{|\alpha|=k}\left\|D^{\alpha} T_{n ; p}^{(m)}(f ; \cdot)\right\|_{\infty, m} \\ \leqslant & 2^{k} C_{2}(p, m)\left\{(n+1)^{m / p} E_{n, \ldots, n}(f)_{p, m}+\sum_{\nu=n+1}^{\infty} \nu^{m / p-1} E_{\nu, \ldots, \nu}(f)_{p, m}\right\} \\ & \quad+m^{k} d^{k} C_{4}(k, p, m) n^{-k} \sum_{\nu=1}^{n} \nu^{k+m / p-1} E_{\nu-1, \ldots, \nu-1}(f)_{p, m} \\ \leqslant & 2^{k} C_{2}(p, m) \sum_{\nu=n+1}^{\infty} \nu^{m / p-1} E_{\nu-1, \ldots, \nu-1}(f)_{p, m} \\ & +C_{5}(k, p, m) n^{-k} \sum_{\nu=1}^{n} \nu^{k+m / p-1} E_{\nu-1, \ldots, \nu-1}(f)_{p, m},\end{aligned}$

где $C_{5}(k, p, m)=2^{k+m / p}(k+m / p) C_{2}(p, m)+m^{k} d^{k} C_{4}(k, p, m)$.

Лемма 1 доказана. 
Лемма 2. Пусть $k \in \mathbb{N}, g \in C(\mathbb{T}), g_{ \pm}(y)=\{g(y) \pm g(-y)\} / 2 u$

$$
g(y) \sim \frac{1}{2} a_{0}(g)+\sum_{n=1}^{\infty}\left(a_{n}(g) \cos n y+b_{n}(g) \sin n y\right)
$$

где $a_{n}(g) \geqslant 0, b_{n}(g) \geqslant 0$. Тогда

1) справедлива оченка

$$
\omega_{k}\left(g ; \frac{\pi}{n}\right)_{\infty, 1} \geqslant \omega_{k}\left(g_{+} ; \frac{\pi}{n}\right)_{\infty, 1} \geqslant C_{6}(k) \sum_{\nu=n}^{\infty} a_{\nu}(g), \quad n \in \mathbb{N}
$$

2) справедлива оченка

$$
\omega_{k}\left(g ; \frac{\pi}{n}\right)_{\infty, 1} \geqslant \omega_{k}\left(g_{+} ; \frac{\pi}{n}\right)_{\infty, 1} \geqslant C_{7}(k) n^{-\varkappa} \sum_{\nu=1}^{n} \nu^{\varkappa} a_{\nu}(g), \quad n \in \mathbb{N}
$$

əде

$$
\varkappa=k+\frac{\left[1-(-1)^{k}\right]}{2}= \begin{cases}k, & k \text { четное }, \\ k+1, & k \text { нечетное }\end{cases}
$$

3) справедлива оченка

$$
\omega_{k}\left(g ; \frac{\pi}{n}\right)_{\infty, 1} \geqslant \omega_{k}\left(g-; \frac{\pi}{n}\right)_{\infty, 1} \geqslant C_{8}(k) n^{-\varkappa} \sum_{\nu=1}^{n} \nu^{\varkappa} b_{\nu}(g), \quad n \in \mathbb{N}
$$

əде

$$
\varkappa=k+\frac{\left[1+(-1)^{k}\right]}{2}= \begin{cases}k+1, & k \text { четное }, \\ k, & k \text { нечетное. }\end{cases}
$$

ДоКАЗАТЕЛЬСтво неравенств 1)-3) приведено в [7, неравенства (22)-(24), с. 72]. Ранее неравенство 1) и неравенство 2) при четном $k$ для симметрических модулей гладкости другим способом доказаны в [8, с. 84-85], [9].

Лемма 3. Пусть $\varepsilon=\left\{\varepsilon_{n}\right\} \in \mathscr{M}_{0}, k \in \mathbb{N}, m \in \mathbb{N}, m<p<\infty, \Delta \varepsilon_{n}=\varepsilon_{n}-\varepsilon_{n+1}$. Тогда последовательность

$$
a_{n}(p ; m ; \varepsilon)=\sum_{\nu=n}^{\infty} \nu^{m / p-1} \Delta \varepsilon_{\nu}, \quad n=1,2, \ldots
$$

обладает следующими свойствами:

1) $0<a_{n}(p ; m ; \varepsilon) \downarrow 0, n \uparrow \infty$;

2) $a_{n}(p ; m ; \varepsilon) \leqslant n^{m / p-1} \varepsilon_{n}$;

3) $\Delta a_{n}(p ; m ; \varepsilon)=a_{n}(p ; m ; \varepsilon)-a_{n+1}(p ; m ; \varepsilon)=n^{m / p-1} \Delta \varepsilon_{n}$; 
4) выполнень оченки

$$
\begin{aligned}
& \varepsilon_{n}=\sum_{\nu=n}^{\infty}\left[(\nu+1)^{1-m / p}-\nu^{1-m / p}\right] a_{\nu+1}(p ; m ; \varepsilon)+n^{1-m / p} a_{n}(p ; m ; \varepsilon), \\
& \varepsilon_{n} \leqslant\left(1-\frac{m}{p}\right) \sum_{\nu=n}^{\infty} \nu^{-m / p} a_{\nu}(p ; m ; \varepsilon)+n^{1-m / p} a_{n}(p ; m ; \varepsilon)
\end{aligned}
$$

5) справедлива оченка

$$
\sum_{n=1}^{\infty} a_{n}(p ; m ; \varepsilon) \leqslant \sum_{n=1}^{\infty} n^{m / p-1} \varepsilon_{n} \leqslant \frac{p}{m} \sum_{n=1}^{\infty} a_{n}(p ; m ; \varepsilon)
$$

6) справедлива оченка

$$
\sum_{\nu=n+1}^{\infty} a_{\nu}(p ; m ; \varepsilon) \leqslant \sum_{\nu=n+1}^{\infty} \nu^{m / p-1} \varepsilon_{\nu} \leqslant \frac{p}{m} \sum_{\nu=n+1}^{\infty} a_{\nu}(p ; m ; \varepsilon)
$$

7) справедлива оченка

$$
\begin{aligned}
n^{-k} \sum_{\nu=1}^{n} \nu^{k} a_{\nu}(p ; m ; \varepsilon) & \leqslant n^{-k} \sum_{\nu=1}^{n} \nu^{k+m / p-1} \varepsilon_{\nu} \\
& \leqslant\left(2-\frac{m}{p}\right) n^{-k} \sum_{\nu=1}^{n} \nu^{k} a_{\nu}(p ; m ; \varepsilon)+\left(1-\frac{m}{p}\right) \sum_{\nu=n+1}^{\infty} a_{\nu}(p ; m ; \varepsilon)
\end{aligned}
$$

ДокАЗАТЕЛЬСтво. Доказательство леммы 3 в случае $m=1$ приведено автором в [1, с. 55-56], в случае $m>1$ доказательство проводится аналогично: 1)-3) проверяются непосредственно; равенство в 4) получается применением преобразования Абеля в представлении

$$
\varepsilon_{n}=\sum_{\nu=n}^{\infty} \Delta \varepsilon_{\nu}=\sum_{\nu=n}^{\infty} \nu^{1-m / p} \Delta a_{\nu}(p ; m ; \varepsilon)
$$

с учетом неравенства 2$)$ и условия $\varepsilon_{n} \downarrow 0, n \uparrow \infty$, а неравенство в 4$)$ следует из полученного равенства в силу оценки $(\nu+1)^{1-m / p}-\nu^{1-m / p} \leqslant(1-m / p) \nu^{-m / p}, \nu=1,2, \ldots$; левые оценки в 5), 6) и 7) имеют место в силу неравенства 2), а правые оценки в 5), 6) и 7) получаются применением неравенства из 4) с последуюшим изменением порядков суммирования в образующихся двойных рядах.

Лемма 4. Пусть $\varepsilon=\left\{\varepsilon_{n}\right\} \in \mathscr{M}_{0}, k \in \mathbb{N}, m \in \mathbb{N}, \Delta \varepsilon_{n}=\varepsilon_{n}-\varepsilon_{n+1}$. Тогда последовательность

$$
a_{n}(m ; \varepsilon)=\sum_{\nu=n}^{\infty}\left(1-\frac{n}{\nu+1}\right)(\nu+1)^{m-1} \Delta \varepsilon_{\nu}, \quad n=1,2, \ldots
$$


обладает следующими свойствами:

1) $0<a_{n}(m ; \varepsilon) \downarrow(n \uparrow)$, при этом $a_{n}(1 ; \varepsilon) \rightarrow 0, n \rightarrow \infty, u a_{n}(m ; \varepsilon) \rightarrow 0, n \rightarrow \infty$, в случае $m>1$ при условии сходимости ряда $\sum_{n=1}^{\infty} n^{m-1} \varepsilon_{n}<+\infty$;

2) $a_{n}(m ; \varepsilon) \leqslant \sum_{\nu=n}^{\infty}(\nu+1)^{m-1} \Delta \varepsilon_{\nu} ;$ в частности, $a_{n}(1 ; \varepsilon) \leqslant \varepsilon_{n}$ nри $m=1$;

3) $\Delta a_{n}(m ; \varepsilon)=a_{n}(m ; \varepsilon)-a_{n+1}(m ; \varepsilon)=\sum_{\nu=n}^{\infty}(\nu+1)^{m-2} \Delta \varepsilon_{\nu} ;$ в частности,

$$
\Delta a_{n}(m ; \varepsilon)= \begin{cases}\sum_{\nu=n}^{\infty}(\nu+1)^{-1} \Delta \varepsilon_{\nu} \leqslant(n+1)^{-1} \varepsilon_{n} & \text { npu } m=1, \\ \sum_{\nu=n}^{\infty} \Delta \varepsilon_{\nu}=\varepsilon_{n} & \text { npu } m=2, \\ \sum_{\nu=n}^{\infty}(\nu+1)^{m-2} \Delta \varepsilon_{\nu} \geqslant(n+1)^{m-2} \varepsilon_{n} & \text { npu } m>2 ;\end{cases}
$$

4) $\Delta^{2} a_{n}(m ; \varepsilon)=\Delta a_{n}(m ; \varepsilon)-\Delta a_{n+1}(m ; \varepsilon)=(n+1)^{m-2} \Delta \varepsilon_{n}$;

5) $a_{n}(m ; \varepsilon)+n \Delta a_{n}(m ; \varepsilon)=\sum_{\nu=n}^{\infty}(\nu+1)^{m-1} \Delta \varepsilon_{\nu} ;$ в частности,

$$
\begin{aligned}
a_{n}(1 ; \varepsilon)+n \Delta a_{n}(1 ; \varepsilon) & =\varepsilon_{n} & & \text { nрu } m=1, \\
a_{n}(m ; \varepsilon)+n \Delta a_{n}(m ; \varepsilon) & \geqslant(n+1)^{m-1} \varepsilon_{n} & & \text { nрu } m>1 ;
\end{aligned}
$$

6) выполнена оценка

$$
\left(m 2^{m-1}\right)^{-1} \sum_{n=1}^{\infty} a_{n}(m ; \varepsilon) \leqslant \sum_{n=1}^{\infty} n^{m-1} \varepsilon_{n} \leqslant 2 \sum_{n=1}^{\infty} a_{n}(m ; \varepsilon)
$$

7) выполнена оценка

$$
\sum_{\nu=n+1}^{\infty} \nu^{m-1} \varepsilon_{\nu} \leqslant 2 \sum_{\nu=n+1}^{\infty} a_{\nu}(m ; \varepsilon)+n a_{n+1}(m ; \varepsilon) ;
$$

8) выполнена оченка

$$
n^{-k} \sum_{\nu=1}^{n} \nu^{k+m-1} \varepsilon_{\nu} \leqslant(k+2) n^{-k} \sum_{\nu=1}^{n} \nu^{k} a_{\nu}(m ; \varepsilon) .
$$

ДокаЗАТЕльство. Доказательство леммы 4 в случае $m=1$ приведено в [10] (см. там лемму 1 при $p=1$ ), она сформулирована также в [1, лемма 2, с. 54]. Отметим, что последовательность $\left\{a_{n}(m ; \varepsilon)\right\}$ в случае $m=1$ ранее рассматривалась Гейтом [11, c. 24]; там же отмечены свойства 1$)-5)$ этой последовательности $\left\{a_{n}(1 ; \varepsilon)\right\}$. Приведем доказательство леммы в случае $m>1$ (случай $m=1$ также включается в эту схему). Свойства 3)-5) проверяются непосредственно, свойство 2) сразу следует из определения $\left\{a_{n}(m ; \varepsilon)\right\}$, а для того чтобы убедиться в справедливости 1$)$, необходимо привлечь свойства 3$)$ и 2 ) в случае $m=1$ и свойства 3 ) и 6 ) в случае $m>1$. Докажем теперь свойства 6), 7) и 8 ). 
6) В силу неравенства в 2) при $m \geqslant 1$ имеем

$$
\begin{aligned}
\sum_{n=1}^{\infty} a_{n}(m ; \varepsilon) & \leqslant \sum_{n=1}^{\infty} \sum_{\nu=n}^{\infty}(\nu+1)^{m-1} \Delta \varepsilon_{\nu}=\sum_{\nu=1}^{\infty}(\nu+1)^{m-1} \Delta \varepsilon_{\nu} \sum_{n=1}^{\nu} 1 \\
& \leqslant 2^{m-1} \sum_{\nu=1}^{\infty} \nu^{m} \Delta \varepsilon_{\nu} \leqslant 2^{m-1} m \sum_{\nu=1}^{\infty} \Delta \varepsilon_{\nu} \sum_{n=1}^{\nu} n^{m-1} \\
& =2^{m-1} m \sum_{n=1}^{\infty} n^{m-1} \sum_{\nu=n}^{\infty} \Delta \varepsilon_{\nu}=m 2^{m-1} \sum_{n=1}^{\infty} n^{m-1} \varepsilon_{n}
\end{aligned}
$$

В силу неравенства в 5) при $m \geqslant 1$ получим

$$
\begin{aligned}
\sum_{n=1}^{\infty} n^{m-1} \varepsilon_{n} & \leqslant \sum_{n=1}^{\infty}(n+1)^{m-1} \varepsilon_{n} \leqslant \sum_{n=1}^{\infty} a_{n}(m ; \varepsilon)+\sum_{n=1}^{\infty} n \Delta a_{n}(m ; \varepsilon) \\
& =\sum_{n=1}^{\infty} a_{n}(m ; \varepsilon)+\sum_{\nu=1}^{\infty} \sum_{n=\nu}^{\infty} \Delta a_{n}(m ; \varepsilon)=2 \sum_{n=1}^{\infty} a_{n}(m ; \varepsilon) .
\end{aligned}
$$

7) В силу неравенства в 5) имеем (при $m \geqslant 1$ )

$$
\begin{aligned}
\sum_{\nu=n+1}^{\infty} \nu^{m-1} \varepsilon_{\nu} & \leqslant \sum_{\nu=n+1}^{\infty}(\nu+1)^{m-1} \varepsilon_{\nu} \leqslant \sum_{\nu=n+1}^{\infty} a_{\nu}(m ; \varepsilon)+\sum_{\nu=n+1}^{\infty} \nu \Delta a_{\nu}(m ; \varepsilon) \\
& =\sum_{\nu=n+1}^{\infty} a_{\nu}(m ; \varepsilon)+\sum_{\nu=n+1}^{\infty} \Delta a_{\nu}(m ; \varepsilon)\left\{\sum_{\mu=1}^{n} 1+\sum_{\mu=n+1}^{\nu} 1\right\} \\
& =\sum_{\nu=n+1}^{\infty} a_{\nu}(m ; \varepsilon)+n a_{n+1}(m ; \varepsilon)+\sum_{\mu=n+1}^{\infty} \sum_{\nu=\mu}^{\infty} \Delta a_{\nu}(m ; \varepsilon) \\
& =2 \sum_{\nu=n+1}^{\infty} a_{\nu}(m ; \varepsilon)+n a_{n+1}(m ; \varepsilon)
\end{aligned}
$$

8) В силу неравенства в 5) получим (при $m \geqslant 1)$

$$
\begin{aligned}
\sum_{\nu=1}^{n} \nu^{k+m-1} \varepsilon_{\nu} & \leqslant \sum_{\nu=1}^{n} \nu^{k}(\nu+1)^{m-1} \varepsilon_{\nu} \leqslant \sum_{\nu=1}^{n} \nu^{k} a_{\nu}(m ; \varepsilon)+\sum_{\nu=1}^{n} \nu^{k+1} \Delta a_{\nu}(m ; \varepsilon) \\
& \leqslant \sum_{\nu=1}^{n} \nu^{k} a_{\nu}(m ; \varepsilon)+(k+1) \sum_{\nu=1}^{n} \Delta a_{\nu}(m ; \varepsilon) \sum_{\mu=1}^{\nu} \mu^{k} \\
& =\sum_{\nu=1}^{n} \nu^{k} a_{\nu}(m ; \varepsilon)+(k+1) \sum_{\mu=1}^{n} \mu^{k} \sum_{\nu=\mu}^{n} \Delta a_{\nu}(m ; \varepsilon) \\
& \leqslant(k+2) \sum_{\nu=1}^{n} \nu^{k} a_{\nu}(m ; \varepsilon) .
\end{aligned}
$$

Лемма 4 доказана. 
Определим теперь функции $G(x ; p ; m ; \varepsilon) \in L_{p}\left(\mathbb{T}^{m}\right)$, используемые при доказательстве оценок снизу в (2). Положим при $p=1$

$$
G(x ; 1 ; m ; \varepsilon)=G_{1}(x ; m ; \varepsilon)+G_{2}(x ; m ; \varepsilon),
$$

где

$$
G_{1}(x ; m ; \varepsilon)=\sum_{n=1}^{\infty} \Delta \varepsilon_{n} F_{n}\left(x_{1}\right) \prod_{i=2}^{m} F_{n}\left(x_{i}\right), \quad G_{2}(x ; m ; \varepsilon)=\sum_{n=1}^{\infty} \Delta \varepsilon_{n} \Phi_{n}\left(x_{1}\right) \prod_{i=2}^{m} F_{n}\left(x_{i}\right),
$$

$F_{n}(y)=1 / 2+\sum_{\nu=1}^{n}(1-\nu /(n+1)) \cos \nu y-$ ядро Фейера порядка $(n+1) \in \mathbb{N}, \Phi_{n}(y)-$ полином (см. $[12$, с. 896$])$

$\sin ((l+1) y) F_{l}(y)=\sum_{\nu=1}^{l+1} \frac{\nu}{2(l+1)} \sin \nu y+\sum_{\nu=l+2}^{2 l+1}\left(1-\frac{\nu}{2(l+1)}\right) \sin \nu y, \quad l \in \mathbb{Z}_{+}, \quad y \in \mathbb{R}$,

в котором $l \equiv[(n+1) / 2]-1, n \in \mathbb{N},[t]$ - целая часть числа $t$. Отметим, что приведенные функции $G_{i}(x ; m ; \varepsilon), i=1,2$, в случае $m=1$ ранее применялись Гейтом в [11, c. 24-26] и были использованы автором [1, с. 57-59] для получения оценки снизу в $(2)$ при $p=m=1$. Полагаем при $p>1$ (в случае $m=1$ указанная функция рассмотрена в заметке автора $[1$, с. 59$\left.] ; \beta_{n}(y)=\cos n y+\sin n y, y \in \mathbb{R}, n \in \mathbb{N}\right)$

$$
\begin{aligned}
G(x ; p ; m ; \varepsilon) & \equiv G_{3}(x ; p ; m ; \varepsilon)=\sum_{n=1}^{\infty} n^{m(1 / p-1)} \Delta \varepsilon_{n} \prod_{i=1}^{m} \sum_{\nu=1}^{n} \beta_{\nu}\left(x_{i}\right) \\
& =\sum_{n=1}^{\infty} \beta_{n}\left(x_{1}\right) \sum_{\nu=n}^{\infty} \nu^{m(1 / p-1)} \Delta \varepsilon_{n} \prod_{i=2}^{m} \sum_{j=1}^{\nu} \beta_{j}\left(x_{i}\right), \quad x=\left(x_{1}, \ldots, x_{m}\right) \in \mathbb{T}^{m} .
\end{aligned}
$$

Лемма 5. Пусть $1 \leqslant p<\infty, m \in \mathbb{N}, \varepsilon=\left\{\varepsilon_{n}\right\} \in \mathscr{M}_{0}, k \in \mathbb{N}$. Тогда функиии $G(x ; p ; m ; \varepsilon)$ обладают следующими свойствами:

1) $G \in L_{p}\left(\mathbb{T}^{m}\right) u E_{n-1, \ldots, n-1}(G)_{p, m} \leqslant C_{9}(p, m) \varepsilon_{n}, n \in \mathbb{N}$;

2) имеет место әквивалентность

$$
G \in C\left(\mathbb{T}^{m}\right) \Longleftrightarrow \sum_{n=1}^{\infty} n^{m / p-1} \varepsilon_{n}<\infty
$$

при этом

$$
C_{10}(p, m) \sum_{n=1}^{\infty} n^{m / p-1} \varepsilon_{n} \leqslant\|G(\cdot ; p ; m ; \varepsilon)\|_{\infty, m} \leqslant C_{11}(p, m) \sum_{n=1}^{\infty} n^{m / p-1} \varepsilon_{n}
$$

3) если ряд в правой части 2) сходится, то

$$
\Omega(p, m, k ; \varepsilon) \equiv \sum_{\nu=n+1}^{\infty} \nu^{m / p-1} \varepsilon_{\nu}+n^{-k} \sum_{\nu=1}^{n} \nu^{k+m / p-1} \varepsilon_{\nu} \leqslant C_{12}(k, p, m) \omega_{k}\left(G ; \frac{d}{n}\right)_{\infty, m} .
$$


ДокаЗАТЕЛЬСтво. Пусть $p=1$. В силу известного равенства (см., например, $[12$, c. 138$])\left\|F_{n}(\cdot)\right\|_{1,1}=1$ имеем оценку $\left\|\Phi_{n}(\cdot)\right\|_{1,1} \leqslant 1$. Отсюда, учитывая, что $F_{n}(y)-$ полином степени $n \in \mathbb{N}$, а $\Phi_{n}(y)$ - полином степени $2 l+1 \equiv 2[(n+1) / 2]-1 \leqslant n \in \mathbb{N}$, получим

$$
\begin{aligned}
\|G(\cdot ; 1 ; m ; \varepsilon)\|_{1, m} & \leqslant\left\|G_{1}(\cdot ; m ; \varepsilon)\right\|_{1, m}+\left\|G_{2}(\cdot ; m ; \varepsilon)\right\|_{1, m} \\
& \leqslant \sum_{n=1}^{\infty} \Delta \varepsilon_{n}\left\{\left\|F_{n}\left(x_{1}\right)\right\|_{1,1}+\left\|\Phi_{n}\left(x_{1}\right)\right\|_{1,1}\right\}\left\|\prod_{i=2}^{m} F_{n}\left(x_{i}\right)\right\|_{1, m-1} \\
& \leqslant 2 \sum_{n=1}^{\infty} \Delta \varepsilon_{n}=2 \varepsilon_{1}, \\
E_{n-1, \ldots, n-1}(G)_{1, m} & \leqslant\left\|\sum_{\nu=n}^{\infty} \Delta \varepsilon_{\nu}\left\{F_{\nu}\left(x_{1}\right)+\Phi_{\nu}\left(x_{1}\right)\right\} \prod_{i=2}^{m} F_{\nu}\left(x_{i}\right)\right\|_{1, m} \\
& \leqslant \sum_{\nu=n}^{\infty} \Delta \varepsilon_{\nu}\left\{\left\|F_{\nu}\left(x_{1}\right)\right\|_{1,1}+\left\|\Phi_{\nu}\left(x_{1}\right)\right\|_{1,1}\right\}\left\|\prod_{i=2}^{m} F_{\nu}\left(x_{i}\right)\right\|_{1, m-1} \\
& \leqslant 2 \sum_{\nu=n}^{\infty} \Delta \varepsilon_{\nu}=2 \varepsilon_{n} .
\end{aligned}
$$

Следовательно, $G(x ; 1 ; m ; \varepsilon) \in L_{1}\left(\mathbb{T}^{m}\right)$ и $E_{n-1, \ldots, n-1}(G)_{1, m} \leqslant 2 \varepsilon_{n}, n \in \mathbb{N}$, т.е. имеет место 1).

Далее, если $\sum_{n=1}^{\infty} n^{m-1} \varepsilon_{n}<\infty$, то из оценки $\left(0<\varepsilon_{n} \downarrow 0\right.$ при $\left.n \uparrow \infty\right)$

$$
\begin{aligned}
& \sum_{n=1}^{\infty} \Delta \varepsilon_{n}\left\|\left\{F_{n}\left(x_{1}\right)+\Phi_{n}\left(x_{1}\right)\right\} \prod_{i=2}^{m} F_{n}\left(x_{i}\right)\right\|_{\infty, m} \\
& \quad \leqslant \sum_{n=1}^{\infty} \Delta \varepsilon_{n}\left\{\frac{n+1}{2}+\frac{1}{2}\left[\frac{n+1}{2}\right]\right\}\left(\frac{n+1}{2}\right)^{m-1} \leqslant \frac{3}{2} \sum_{n=1}^{\infty}\left(\frac{n+1}{2}\right)^{m} \Delta \varepsilon_{n} \\
& \quad \leqslant \frac{3}{2} \sum_{n=1}^{\infty} n^{m} \Delta \varepsilon_{n} \leqslant \frac{3 m}{2} \sum_{n=1}^{\infty} \Delta \varepsilon_{n} \sum_{\nu=1}^{n} \nu^{m-1} \\
& \quad=\frac{3 m}{2} \sum_{\nu=1}^{\infty} \nu^{m-1} \sum_{n=\nu}^{\infty} \Delta \varepsilon_{n}=\frac{3 m}{2} \sum_{\nu=1}^{\infty} \nu^{m-1} \varepsilon_{\nu}
\end{aligned}
$$

в силу известного признака равномерной сходимости Вейерштрасса следует, что функция $G(x ; 1 ; m ; \varepsilon) \in C\left(\mathbb{T}^{m}\right)$ и справедлива правая оценка в 2$)$ с постоянной $C_{11}(1, m)=$ $3 m / 2$. С другой стороны, если $G(x ; 1 ; m ; \varepsilon) \in C\left(\mathbb{T}^{m}\right)$, то

$$
\begin{aligned}
\|G(\cdot ; 1 ; m ; \varepsilon)\|_{\infty, m} & \geqslant|G((0, \ldots, 0) ; 1 ; m ; \varepsilon)|=\left|\sum_{n=1}^{\infty} \Delta \varepsilon_{n}\left\{F_{n}(0)+\Phi_{n}(0)\right\} \prod_{i=2}^{m} F_{n}(0)\right| \\
& =\sum_{n=1}^{\infty} \Delta \varepsilon_{n}\left(\frac{n+1}{2}\right)^{m} \geqslant 2^{-m} \sum_{n=1}^{\infty} n^{m} \Delta \varepsilon_{n} \geqslant 2^{-m} \sum_{n=1}^{\infty} \Delta \varepsilon_{n} \sum_{\nu=1}^{n} \nu^{m-1} \\
& =2^{-m} \sum_{\nu=1}^{\infty} \nu^{m-1} \sum_{n=\nu}^{\infty} \Delta \varepsilon_{n}=2^{-m} \sum_{\nu=1}^{\infty} \nu^{m-1} \varepsilon_{\nu},
\end{aligned}
$$


откуда следует сходимость ряда $\sum_{n=1}^{\infty} n^{m-1} \varepsilon_{n}<\infty$ и справедливость левой оценки в 2) с постоянной $C_{10}(1, m)=2^{-m}$.

Перейдем к доказательству 3$)$. Если ряд в правой части 2$)$ сходится, то $G(x ; 1 ; m ; \varepsilon) \in$ $C\left(\mathbb{T}^{m}\right)$. Обозначим

$$
\begin{aligned}
g(y ; 1 ; m ; \varepsilon) & \equiv G((y, 0, \ldots, 0) ; 1 ; m ; \varepsilon)=G_{1}((y, 0, \ldots, 0) ; m ; \varepsilon)+G_{2}((y, 0, \ldots, 0) ; m ; \varepsilon) \\
& \equiv g_{1}(y ; m ; \varepsilon)+g_{2}(y ; m ; \varepsilon), \quad y \in \mathbb{R} ;
\end{aligned}
$$

очевидно, что

$$
\begin{aligned}
& g_{1}(y ; m ; \varepsilon)=\frac{1}{2}\{g(y ; 1 ; m ; \varepsilon)+g(-y ; 1 ; m ; \varepsilon)\} \\
& g_{2}(y ; m ; \varepsilon)=\frac{1}{2}\{g(y ; 1 ; m ; \varepsilon)-g(-y ; 1 ; m ; \varepsilon)\}
\end{aligned}
$$

откуда $g_{i}(y ; m ; \varepsilon) \in C(\mathbb{T})$ и $\omega_{k}\left(g_{i} ; \delta\right)_{\infty, 1} \leqslant \omega_{k}(g ; \delta)_{\infty, 1}, i=1,2$. Далее, имеем

$$
\begin{aligned}
g_{1}(y ; m ; \varepsilon)= & \sum_{n=1}^{\infty} \Delta \varepsilon_{n} F_{n}(y)\left(\frac{n+1}{2}\right)^{m-1} \\
= & 2^{-m+1} \sum_{n=1}^{\infty}(n+1)^{m-1} \Delta \varepsilon_{n}\left\{\frac{1}{2}+\sum_{\nu=1}^{n}\left(1-\frac{\nu}{n+1}\right) \cos \nu y\right\} \\
= & 2^{-m} \sum_{n=1}^{\infty}(n+1)^{m-1} \Delta \varepsilon_{n} \\
& +2^{-(m-1)} \sum_{n=1}^{\infty}\left\{\sum_{\nu=n}^{\infty}(\nu+1)^{m-1} \Delta \varepsilon_{\nu}\left(1-\frac{n}{\nu+1}\right)\right\} \cos n y \\
\equiv & \frac{1}{2} a_{0}\left(g_{1}\right)+\sum_{n=1}^{\infty} a_{n}\left(g_{1}\right) \cos n y, \quad y \in \mathbb{R},
\end{aligned}
$$

где $a_{n}\left(g_{1}\right)=2^{-(m-1)} a_{n}(m ; \varepsilon), n \in \mathbb{N}$, а последовательность $\left\{a_{n}(m ; \varepsilon)\right\}$ определена в лемме 4. Так как по условию $\sum_{n=1}^{\infty} n^{m-1} \varepsilon_{n}<\infty$, то в силу 6) леммы 4 выполнена оценка $\sum_{n=1}^{\infty} a_{n}(m ; \varepsilon)<\infty$; отсюда в силу неравенств 7$)$ и 8) леммы 4 и неравенств 1$)$ при $k \in \mathbb{N}$ и 2$)$ при $k$ четном леммы 2 получим $\left(a_{n}(m ; \varepsilon) \downarrow\right.$ при $\left.n \uparrow\right)$

$$
\begin{aligned}
\Omega(1, m, k ; \varepsilon) & \leqslant 2 \sum_{\nu=n+1}^{\infty} a_{\nu}(m ; \varepsilon)+n a_{n+1}(m ; \varepsilon)+(k+2) n^{-k} \sum_{\nu=1}^{n} \nu^{k} a_{\nu}(m ; \varepsilon) \\
& \leqslant 2 \sum_{\nu=n+1}^{\infty} a_{\nu}(m ; \varepsilon)+(2 k+3) n^{-k} \sum_{\nu=1}^{n} \nu^{k} a_{\nu}(m ; \varepsilon) \\
& \leqslant 2 C_{6}^{-1}(k) \omega_{k}\left(g_{1} ; \frac{\pi}{n}\right)_{\infty, 1}+(2 k+3) C_{7}^{-1}(k) \omega_{k}\left(g_{1} ; \frac{\pi}{n}\right)_{\infty, 1} \\
& \leqslant\left\{2 C_{6}^{-1}(k)+(2 k+3) C_{7}^{-1}(k)\right\} \omega_{k}\left(g ; \frac{\pi}{n}\right)_{\infty, 1} .
\end{aligned}
$$


Для завершения доказательства остается оценить второе слагаемоев 3) при нечетном $k$, для чего согласно 3 ) леммы 2 воспользуемся функцией $g_{2}$. Имеем

$$
\begin{aligned}
g_{2}(y ; m ; \varepsilon)= & \sum_{n=1}^{\infty} \Delta \varepsilon_{n} \Phi_{n}(y)\left(\frac{n+1}{2}\right)^{m-1} \\
= & 2^{-m+1} \sum_{n=1}^{\infty}(n+1)^{m-1} \Delta \varepsilon_{n}\left\{\sum_{\nu=1}^{[(n+1) / 2]} \frac{\nu}{2[(n+1) / 2]} \sin \nu y\right. \\
& \left.+\sum_{\nu=[(n+1) / 2]+1}^{2[(n+1) / 2]-1}\left(1-\frac{\nu}{2[(n+1) / 2]}\right) \sin \nu y\right\} \\
= & 2^{-m+1} \sum_{\nu=1}^{\infty} b_{\nu}(m ; \varepsilon) \sin \nu y,
\end{aligned}
$$

где

$$
b_{\nu}(m ; \varepsilon) \equiv \sum_{n=\nu}^{2(\nu-1)}(n+1)^{m-1} \Delta \varepsilon_{n}\left(1-\frac{\nu}{2[(n+1) / 2]}\right)+\nu \sum_{n=2 \nu-1}^{\infty} \frac{(n+1)^{m-1} \Delta \varepsilon_{n}}{2[(n+1) / 2]}
$$

Отсюда в силу неравенства 3) леммы 2 получим

$$
\begin{aligned}
C_{8}^{-1} & (k) n^{k} \omega_{k}\left(g_{2} ; \frac{\pi}{n}\right)_{\infty, 1} \geqslant 2^{-(m-1)} \sum_{\nu=1}^{n} \nu^{k} b_{\nu}(m ; \varepsilon) \\
& \geqslant \sum_{\nu=1}^{n} \nu^{k+1} \sum_{j=2 \nu-1}^{n}\left(\frac{j+1}{2}\right)^{m-1} \Delta \varepsilon_{j}\left(2\left[\frac{j+1}{2}\right]\right)^{-1} \\
& =\sum_{j=1}^{n}\left(\frac{j+1}{2}\right)^{m-1} \Delta \varepsilon_{j}\left(2\left[\frac{j+1}{2}\right]\right)^{-1} \sum_{\nu=1}^{[(j+1) / 2]} \nu^{k+1} \\
& \geqslant \frac{1}{k+2} \sum_{j=1}^{n}\left(\frac{j+1}{2}\right)^{m-1} \Delta \varepsilon_{j}\left(2\left[\frac{j+1}{2}\right]\right)^{-1}\left(\left[\frac{j+1}{2}\right]\right)^{k+2} \\
\geqslant & \frac{1}{2(k+2)} \sum_{j=1}^{n} \Delta \varepsilon_{j}\left(\left[\frac{j+1}{2}\right]\right)^{m+k} \geqslant \frac{2^{-(m+k)}}{2(k+2)} \sum_{j=1}^{n} j^{m+k} \Delta \varepsilon_{j} \\
\geqslant & \frac{2^{-(m+k)}}{2(k+2)} \sum_{j=1}^{n} \Delta \varepsilon_{j} \sum_{\nu=1}^{j} \nu^{m+k-1}=\frac{2^{-(m+k)}}{2(k+2)} \sum_{\nu=1}^{n} \nu^{m+k-1} \sum_{j=\nu}^{n} \Delta \varepsilon_{j} \\
= & \frac{2^{-(m+k)}}{2(k+2)}\left\{\sum_{\nu=1}^{n} \nu^{m+k-1} \varepsilon_{\nu}-\varepsilon_{n+1} \sum_{\nu=1}^{n} \nu^{m+k-1}\right\},
\end{aligned}
$$

и, следовательно,

$$
\begin{aligned}
n^{-k} \sum_{\nu=1}^{n} \nu^{m+k-1} \varepsilon_{\nu} & \leqslant(k+2) 2^{m+k+1} C_{8}^{-1}(k) \omega_{k}\left(g_{2} ; \frac{\pi}{n}\right)_{\infty, 1}+\varepsilon_{n+1} n^{-k} \sum_{\nu=1}^{n} \nu^{m+k-1} \\
& \leqslant(k+2) 2^{m+k+1} C_{8}^{-1}(k) \omega_{k}\left(g_{2} ; \frac{\pi}{n}\right)_{\infty, 1}+n^{m} \varepsilon_{n+1} .
\end{aligned}
$$


Далее, в силу 2) леммы 2 (при $k$ нечетном) и 8 ) леммы 4

$$
\begin{aligned}
C_{7}^{-1}(k) n^{k+1} \omega_{k}\left(g_{1} ; \frac{\pi}{n}\right)_{\infty, 1} & \geqslant \sum_{\nu=1}^{n} \nu^{k+1} a_{\nu}(m ; \varepsilon) \geqslant(k+3)^{-1} \sum_{\nu=1}^{n} \nu^{k+m} \varepsilon_{\nu} \\
& \geqslant(k+3)^{-1}(k+m+1)^{-1} n^{k+m+1} \varepsilon_{n},
\end{aligned}
$$

откуда $n^{m} \varepsilon_{n+1} \leqslant n^{m} \varepsilon_{n} \leqslant(k+3)(k+m+1) C_{7}^{-1}(k) \cdot \omega_{k}\left(g_{1} ; \pi / n\right)_{\infty, 1}$. Объединяя полученные оценки, имеем ( $k$ нечетное)

$$
\begin{aligned}
n^{-k} \sum_{\nu=1}^{n} \nu^{k+m-1} \varepsilon_{\nu} \leqslant & (k+2) 2^{m+k+1} C_{8}^{-1}(k) \omega_{k}\left(g_{2} ; \frac{\pi}{n}\right)_{\infty, 1} \\
& +(k+3)(k+m+1) C_{7}^{-1}(k) \omega_{k}\left(g_{1} ; \frac{\pi}{n}\right)_{\infty, 1} \\
\leqslant & C_{13}(k, m) \omega_{k}\left(g ; \frac{\pi}{n}\right)_{\infty, 1} .
\end{aligned}
$$

Предположим теперь, что $p>1$. В силу известной одномерной оценки (см., например, $[13$, c. 209$],[1$, c. 56$])$

$$
\left\|\sum_{\nu=1}^{n} \beta_{\nu}(\cdot)\right\|_{p, 1} \leqslant C_{14}(p) n^{1-1 / p}
$$

имеем $\left(0<\varepsilon_{n} \downarrow 0\right.$ при $\left.n \uparrow \infty\right)$

$$
\begin{aligned}
\left\|G_{3}\right\|_{p, m} & \leqslant \sum_{n=1}^{\infty} n^{m(1 / p-1)} \Delta \varepsilon_{n}\left\|\prod_{i=1}^{m} \sum_{\nu=1}^{n} \beta_{\nu}\left(x_{i}\right)\right\|_{p, m} \\
& =\sum_{n=1}^{\infty} n^{m(1 / p-1)} \Delta \varepsilon_{n} \prod_{i=1}^{m}\left\|\sum_{\nu=1}^{n} \beta_{\nu}\left(x_{i}\right)\right\|_{p, 1} \\
& \leqslant C_{14}^{m}(p) \sum_{n=1}^{\infty} \Delta \varepsilon_{n}=C_{14}^{m}(p) \varepsilon_{1} \\
E_{n-1, \ldots, n-1}\left(G_{3}\right)_{p, m} & \leqslant\left\|\sum_{\nu=n}^{\infty} \nu^{m(1 / p-1)} \Delta \varepsilon_{\nu} \prod_{i=1}^{m} \sum_{\mu=1}^{\nu} \beta_{\mu}\left(x_{i}\right)\right\|_{p, m} \\
& \leqslant \sum_{\nu=n}^{\infty} \nu^{m(1 / p-1)} \Delta \varepsilon_{\nu}\left\|\prod_{i=1}^{m} \sum_{\mu=1}^{\nu} \beta_{\mu}\left(x_{i}\right)\right\|_{p, m} \\
& \leqslant C_{14}^{m}(p) \sum_{\nu=n}^{\infty} \Delta \varepsilon_{\nu}=C_{14}^{m}(p) \varepsilon_{n}
\end{aligned}
$$

откуда $G_{3}(x ; p ; m ; \varepsilon) \in L_{p}\left(\mathbb{T}^{m}\right)$ и $E_{n-1, \ldots, n-1}\left(G_{3}\right)_{p, m} \leqslant C_{9}(p, m) \varepsilon_{n}, n \in \mathbb{N}$, т.е. имеет место 1).

Далее, если $\sum_{n=1}^{\infty} n^{m / p-1} \varepsilon_{n}<\infty$, то из оценки $\left(0<\varepsilon_{n} \downarrow 0\right.$ при $\left.n \uparrow \infty\right)$

$$
\sum_{n=1}^{\infty} n^{m(1 / p-1)} \Delta \varepsilon_{n}\left\|\prod_{i=1}^{m} \sum_{\nu=1}^{n} \beta_{\nu}\left(x_{i}\right)\right\|_{\infty, m}
$$




$$
\begin{aligned}
& =\sum_{n=1}^{\infty} n^{m(1 / p-1)} \Delta \varepsilon_{n} \prod_{i=1}^{m}\left\|\sum_{\nu=1}^{n} \beta_{\nu}\left(x_{i}\right)\right\|_{\infty, 1} \leqslant(\sqrt{2})^{m} \sum_{n=1}^{\infty} n^{m / p} \Delta \varepsilon_{n} \\
& \leqslant 2^{m / 2} C_{15}\left(\frac{m}{p}\right) \sum_{n=1}^{\infty} \Delta \varepsilon_{n} \sum_{\nu=1}^{n} \nu^{m / p-1}=2^{m / 2} C_{15}\left(\frac{m}{p}\right) \sum_{\nu=1}^{\infty} \nu^{m / p-1} \sum_{n=\nu}^{\infty} \Delta \varepsilon_{n} \\
& =2^{m / 2} C_{15}\left(\frac{m}{p}\right) \sum_{\nu=1}^{\infty} \nu^{m / p-1} \varepsilon_{\nu},
\end{aligned}
$$

где $C_{15}(m / p)=\max \{m / p ; 1\}$, следует в силу признака равномерной сходимости Вейерштрасса, что функция $G_{3}(x ; p ; m ; \varepsilon) \in C\left(\mathbb{T}^{m}\right)$ и справедлива правая оценка в 2) с постоянной $C_{11}(p, m)=2^{m / 2} C_{15}(m / p)$. С другой стороны, если $G_{3} \in C\left(\mathbb{T}^{m}\right)$, то $\left(0<\varepsilon_{n} \downarrow 0\right.$ при $n \uparrow \infty)$

$$
\begin{aligned}
\sum_{n=1}^{\infty} n^{m / p-1} \varepsilon_{n} & =\sum_{n=1}^{\infty} n^{m / p-1} \sum_{\nu=n}^{\infty} \Delta \varepsilon_{\nu}=\sum_{\nu=1}^{\infty} \Delta \varepsilon_{\nu} \sum_{n=1}^{\nu} n^{m / p-1} \\
& \leqslant C_{16}\left(\frac{m}{p}\right) \sum_{\nu=1}^{\infty} \nu^{m / p} \Delta \varepsilon_{\nu}=C_{16}\left(\frac{m}{p}\right) \sum_{\nu=1}^{\infty} \nu^{m(1 / p-1)} \Delta \varepsilon_{\nu} \nu^{m} \\
& =C_{16}\left(\frac{m}{p}\right) \sum_{\nu=1}^{\infty} \nu^{m(1 / p-1)} \Delta \varepsilon_{\nu} \prod_{i=1}^{m} \sum_{\mu=1}^{\nu} \beta_{\mu}(0) \\
& =C_{16}\left(\frac{m}{p}\right) G_{3}((0, \ldots, 0) ; p ; m ; \varepsilon) \\
& \leqslant C_{16}\left(\frac{m}{p}\right)\left\|G_{3}(\cdot ; p ; m ; \varepsilon)\right\|_{\infty, m}<\infty
\end{aligned}
$$

где

$$
C_{16}\left(\frac{m}{p}\right)=\left\{\begin{array}{cc}
\frac{p}{m} & \text { при } \frac{m}{p} \leqslant 1, \\
1 & \text { при } \frac{m}{p} \geqslant 1 .
\end{array}\right.
$$

Докажем 3). Если ряд в правой части 2$)$ сходится, то $G_{3}(x ; p ; m ; \varepsilon) \in C\left(\mathbb{T}^{m}\right)$. Обозначим

$$
\begin{aligned}
g_{3}(y ; p ; m ; \varepsilon) & \equiv G_{3}((y, 0, \ldots, 0) ; p ; m ; \varepsilon) \\
& =\sum_{n=1}^{\infty} n^{m / p-1} \Delta \varepsilon_{n} \sum_{\nu=1}^{n} \beta_{\nu}(y)=\sum_{n=1}^{\infty} a_{n}(p ; m ; \varepsilon) \beta_{n}(y), \quad y \in \mathbb{R},
\end{aligned}
$$

где

$$
a_{n}(p ; m ; \varepsilon)=\sum_{\nu=n}^{\infty} \nu^{m / p-1} \Delta \varepsilon_{\nu}, \quad n \in \mathbb{N} .
$$

Отметим, что ряд $\sum_{n=1}^{\infty} a_{n}(p ; m ; \varepsilon)<\infty$ (см. доказательство 2$) ;$ в случае $m / p<1$ сходимость указанного ряда имеет место в силу левого неравенства в 5) леммы 3 ). В 
случае $m / p<1$ в силу правых неравенств в 6) и 7) леммы 3 и неравенств 1$), 2)$ при четном $k$, либо неравенства 3$)$ при нечетном $k$ из леммы 2 имеем

$$
\begin{aligned}
& \Omega(p, m, k ; \varepsilon) \leqslant\left(1-\frac{m}{p}+\frac{p}{m}\right) \sum_{\nu=n+1}^{\infty} a_{\nu}(p ; m ; \varepsilon)+\left(2-\frac{m}{p}\right) n^{-k} \sum_{\nu=1}^{n} \nu^{k} a_{\nu}(p ; m ; \varepsilon) \\
& \quad \leqslant\left(1-\frac{m}{p}+\frac{p}{m}\right) C_{6}^{-1}(k) \omega_{k}\left(\left(g_{3}\right)_{+} ; \frac{\pi}{n}\right)_{\infty, 1}+\left(2-\frac{m}{p}\right) C_{17}(k) \omega_{k}\left(\varphi ; \frac{\pi}{n}\right)_{\infty, 1} \\
& \quad \leqslant C_{18}(k, p, m) \omega_{k}\left(g_{3} ; \frac{\pi}{n}\right)_{\infty, 1},
\end{aligned}
$$

где $\varphi \equiv\left(g_{3}\right)_{+}$и $C_{17}(k)=C_{7}^{-1}(k)$ при четном $k, \varphi \equiv\left(g_{3}\right)_{-}$и $C_{17}(k)=C_{8}^{-1}(k)$ при нечетном $k,\left(g_{3}\right)_{ \pm}(y)=(1 / 2)\left\{g_{3}(y) \pm g_{3}(-y)\right\}$. В случае $m / p \geqslant 1$ в силу очевидной оценки $a_{n}(p ; m ; \varepsilon) \geqslant n^{m / p-1} \varepsilon_{n}$ аналогично получим

$$
\begin{aligned}
& \Omega(p, m, k ; \varepsilon) \leqslant \sum_{\nu=n+1}^{\infty} a_{\nu}(p ; m ; \varepsilon)+n^{-k} \sum_{\nu=1}^{n} \nu^{k} a_{\nu}(p ; m ; \varepsilon) \\
& \leqslant C_{6}^{-1}(k) \omega_{k}\left(\left(g_{3}\right)_{+} ; \frac{\pi}{n}\right)_{\infty, 1}+C_{17}(k) \omega_{k}\left(\varphi ; \frac{\pi}{n}\right)_{\infty, 1} \leqslant C_{19}(k) \omega_{k}\left(g_{3} ; \frac{\pi}{n}\right)_{\infty, 1} .
\end{aligned}
$$

Для завершения доказательства 3) (как при $p=1$, так и при $p>1$ ) нужно воспользоваться оценкой $\omega_{k}(g ; \pi / n)_{\infty, 1} \leqslant \omega_{k}(G ; d / n)_{\infty, m}$, которая следует из цепочки неравенств

$$
\begin{aligned}
\left|\Delta_{\eta}^{k} g(y ; p ; m ; \varepsilon)\right| & =\left|\Delta_{1, \eta}^{k} G((y, 0, \ldots, 0) ; p ; m ; \varepsilon)\right| \leqslant\left\|\Delta_{1, \eta}^{k} G(\cdot ; p ; m ; \varepsilon)\right\|_{\infty, m} \\
& \leqslant \omega_{k}^{(1)}\left(G ; \frac{\pi}{n}\right)_{\infty, m} \leqslant \omega_{k}\left(G ; \frac{\pi}{n}, \ldots, \frac{\pi}{n}\right)_{\infty, m} \leqslant \omega_{k}\left(G ; \frac{d}{n}\right)_{\infty, m}
\end{aligned}
$$

$\forall y \in \mathbb{R}, \quad \forall \eta \in \mathbb{R}, \quad|\eta| \leqslant \frac{\pi}{n}$,

где $\omega_{k}^{(1)}(G ; \pi / n)_{\infty, m}$ и $\omega_{k}(G ; \pi / n, \ldots, \pi / n)_{\infty, m}-$ соответственно частньй модуль гладкости по первой переменной и полньй кубический модуль гладкости $k$-го порядка функции $G \in C\left(\mathbb{T}^{m}\right)$ (см., например, [3, с. 123-127]).

Лемма 5 доказана.

ЗАмЕчАниЕ. В процессе доказательства леммы 5 для функций $G(x ; p ; m ; \varepsilon) \in$ $L_{p}\left(\mathbb{T}^{m}\right)$ в точке $x=0$ были получены оценки: при $p=1$

$$
G((0, \ldots, 0) ; 1 ; m ; \varepsilon)=g_{1}(0 ; 1 ; m ; \varepsilon) \geqslant 2^{-m} \sum_{n=1}^{\infty} n^{m-1} \varepsilon_{n}
$$

при $p>1$

$$
G((0, \ldots, 0) ; p ; m ; \varepsilon)=g_{3}(0 ; p ; m ; \varepsilon) \geqslant C_{16}^{-1}\left(\frac{m}{p}\right) \sum_{n=1}^{\infty} n^{m / p-1} \varepsilon_{n}
$$


из которых, в частности, следует, что если ряд

$$
\sum_{n=1}^{\infty} n^{m / p-1} \varepsilon_{n}=\infty, \quad p \geqslant 1
$$

то $G(x ; p ; m ; \varepsilon)$ является существенно неограниченной на $\mathbb{T}^{m}=[-\pi, \pi]^{m}($ точнее, в любой окрестности начала координат). Для доказательства последнего утверждения предположим противное (здесь мы следуем схеме рассуждений, приведенной в $[13$, c. 210-211] в случае $m=1$ и распространенной на случай $m>1$ в [4, с. 145-146], [14, c. 109-110]). То есть, мы предполагаем, что имеет место эквивалентность $G(x ; p ; m ; \varepsilon)$ некоторой функции, ограниченной на $\mathbb{T}^{m}$, и, следовательно, в этом случае

$$
\operatorname{ess} \sup \left\{|G(x ; p ; m ; \varepsilon)| ; x \in \mathbb{T}^{m}\right\} \leqslant B<\infty \text {. }
$$

Но тогда (см., например, [12, с. 141], [14, с. 109])

$$
\operatorname{ess} \sup \left\{\left|\sigma_{n}^{(m)}(G ; x)\right| x \in \mathbb{T}^{m}\right\} \leqslant B, \quad n \in \mathbb{Z}_{+},
$$

где $\sigma_{n}^{(m)}(G ; x) \equiv \sigma_{n, \ldots, n}\left(G ; x_{1}, \ldots, x_{m}\right)$ - средние Фейера порядка $n \in \mathbb{Z}_{+}$по переменной $x_{i}, i=\overline{1, m}$ (или средние арифметические частных сумм), ряда Фурье функции $G \in L_{p}\left(\mathbb{T}^{m}\right)$. Последнее неравенство противоречит оценкам (8) и $(9)$, ибо метод средних арифметических является вполне регулярньм методом суммирования (см. [12, с. 27, замечание 1]). Действительно, при $p=1$

$$
\begin{aligned}
0 & \leqslant S_{n}^{(m)}(G ; 0)=S_{n}^{(1)}\left(g_{1} ; 0\right)=\frac{1}{2} a_{0}\left(g_{1}\right)+\sum_{\nu=1}^{n} a_{\nu}\left(g_{1}\right) \\
& \geqslant 2^{-(m-1)} \sum_{\nu=1}^{n} a_{\nu}(m ; \varepsilon) \geqslant 2^{-m} \sum_{\nu=1}^{n} \nu^{m-1} \varepsilon_{\nu} \rightarrow \infty \quad \text { при } n \rightarrow \infty
\end{aligned}
$$

(здесь $S_{n}^{(m)}(G ; x) \equiv S_{n, \ldots, n}\left(G ; x_{1}, \ldots, x_{m}\right)$ - частная сумма порядка $n \in \mathbb{Z}_{+}$по переменной $x_{i}, i=\overline{1, m}$, ряда $Ф$ урье функции $\left.G\right)$, а при $p>1$

$$
\begin{aligned}
0 & \leqslant S_{n}^{(m)}(G ; 0)=S_{n}^{(1)}\left(g_{3} ; 0\right)=\sum_{\nu=1}^{n} a_{\nu}(p ; m ; \varepsilon)=\sum_{\nu=1}^{n} \nu^{m / p} \Delta \varepsilon_{\nu} \\
& \geqslant C_{16}^{-1}\left(\frac{m}{p}\right) \sum_{\nu=1}^{n} \nu^{m / p-1} \varepsilon_{\nu} \rightarrow \infty \quad \text { при } n \rightarrow \infty .
\end{aligned}
$$

Поэтому

$$
\lim _{n \rightarrow \infty} \sigma_{n}^{(m)}(G ; 0)=\infty
$$

что противоречит $\sup \left\{\left|\sigma_{n}^{(m)}(G ; 0)\right| ; n \in \mathbb{N}\right\}<\infty$. 
ДоКАЗАТЕЛЬСТВО ТЕОРЕМЫ. Условие сходимости ряда в (1) необходимо и достаточно для того, чтобы любая функция $f \in L_{p}\left(\mathbb{T}^{m}\right)$, для которой имеет место

$$
E_{n-1, \ldots, n-1}(f)_{p, m}=O\left(\varepsilon_{n}\right), \quad n \uparrow \infty
$$

(в частности, каждая функция $f \in E_{p, m}[\varepsilon]$ ), была эквивалентна некоторой функции $\psi \in C\left(\mathbb{T}^{m}\right)$. Достаточность известна (см. первую часть утверждения леммы 1 ) и следует из соответствующих результатов [2, с. 56], [3, с. 378] в случае $m=1$ и [4, с. 141], $[15$, с. 88-89] в случае $m>1$. Необходимость также известна: если ряд в (1) расходится, то существует функция $G(x ; p ; m ; \varepsilon) \in L_{p}\left(\mathbb{T}^{m}\right)$ с $E_{n-1, \ldots, n-1}(G)_{p, m}=O\left(\varepsilon_{n}\right)$, $n \in \mathbb{N}$, которая является существенно неограниченной в окрестности начала координат, и, следовательно, на $\mathbb{T}^{m}=[-\pi, \pi]^{m}$ (ясно, что такая функция не может быть эквивалентной никакой функции $\left.\Psi \in C\left(\mathbb{T}^{m}\right)\right)$. Последнее утверждение при $m=1$ доказано в [16, с. 214-215] (см. также [13, с. 210-211], [1, с. 57-59] и замечание после леммы 5 настоящей заметки); при $m>1$ оно отмечено в [15, с. 89], а доказательство фактически приведено в $[4$, с. 144-146], [14, с. 108-111].

Оценка сверху в (2) следует из неравенства (4) леммы 1: если произвольная функция $f$ принадлежит классу $E_{p, m}[\varepsilon]$ и сходится ряд в $(1)$, то в силу леммы $1 f$ эквивалентна некоторой функции $\psi \in C\left(\mathbb{T}^{m}\right)$ и справедлива оценка

$$
\begin{aligned}
\omega_{k}\left(\psi ; \frac{d}{n}\right)_{\infty, m} \leqslant & C_{1}(k, p, m)\left\{\sum_{\nu=n+1}^{\infty} \nu^{m / p-1} E_{\nu-1, \ldots, \nu-1}(f)_{p, m}\right. \\
& \left.+n^{-k} \sum_{\nu=1}^{n} \nu^{k+m / p-1} E_{\nu-1, \ldots, \nu-1}(f)_{p, m}\right\} \\
\leqslant & C_{1}(k, p, m)\left\{\sum_{\nu=n+1}^{\infty} \nu^{m / p-1} \varepsilon_{\nu}+n^{-k} \sum_{\nu=1}^{n} \nu^{k+m / p-1} \varepsilon_{\nu}\right\} .
\end{aligned}
$$

Оценка снизу в (2) реализуется с помощью функции $C_{9}^{-1}(p, m) G(x ; p ; m ; \varepsilon) \in E_{p, m}[\varepsilon]$, где $C_{9}(p, m)$ - постоянная из 1$)$ леммы 5 . А именно, в силу 3$)$ леммы 5 имеем

$$
\begin{aligned}
& \sum_{\nu=n+1}^{\infty} \nu^{m / p-1} \varepsilon_{\nu}+n^{-k} \sum_{\nu=1}^{n} \nu^{k+m / p-1} \varepsilon_{\nu} \leqslant C_{12}(k, p, m) \omega_{k}\left(G ; \frac{d}{n}\right)_{\infty, m} \\
& \leqslant C_{12}(k, p, m) C_{9}(p, m) \sup \left\{\omega_{k}\left(\psi ; \frac{d}{n}\right)_{\infty, m} ; f \in E_{p, m}[\varepsilon]\right\} .
\end{aligned}
$$

\section{СПИСОК ЦИТИРОВАННОЙ ЛИТЕРАТУРЫ}

[1] Ильясов Н. А. К обратной теореме теории приближений периодических функций в разных метриках // Матем. заметки. 1992. Т. 52. № 2. С. 53-61.

[2] Конюшков А. А. Наилучшие приближения тригонометрическими полиномами и коэффициенты Фурье // Матем. сб. 1958. Т. 44. № 1. С. 53-84.

[3] Тиман А.Ф. Теория приближения функций действительного переменного. М.: Физматгиз, 1960.

[4] Темиргалиев Н. Т. О связи теорем вложения с равномерной сходимостью кратных рядов Фурье // Матем. заметки. 1972. Т. 12. № 2. С. 139-148. 
[5] Никольский С. М. Приближение функций многих переменных и теоремы вложения. М.: Наука, 1969.

[6] Стечкин С.Б. О порядке наилучших приближений непрерьвных функций // Изв. АН СССР. Сер. матем. 1951. Т. 15. №3. С. 219-242.

[7] Гейт В.Э. Теоремы вложения для некоторых классов периодических непрерывных функций // Изв. вузов. Матем. 1972. № 4. С. 67-77.

[8] Жук В. В. Об одном методе суммирования рядов Фурье. Ряды Фурье с положительньми коэффициентами // Сб. научных трудов Ленингр. мех. ин-та. 1965. № 50. С. 73-92.

[9] Boas R. P. Fourier series with positive coefficients // J. Math. Anal. Appl. 1967. V. 17. №3. P. 463-483.

[10] Ильясов Н. А. О приближении периодических функций средними Фейера-Зигмунда в разных метриках // Матем. заметки. 1990. Т. 48. № 4. С. 48-57.

[11] Гейт В.Э. О структурных и конструктивных свойствах функции и ее сопряженной в $L / /$ Изв. вузов. Матем. 1972. №7. С. 19-30.

[12] Бари Н. К. Тригонометрические ряды. М.: Физматгиз, 1961.

[13] Ульянов П. Л. Об абсолютной и равномерной сходимости рядов Фурье // Матем. сб. 1967. T. 72. № 2. C. 193-225.

[14] Темиргалиев Н.Т. Об одной теореме вложения // Изв. вузов. Матем. 1973. № 7. C. 103-111.

[15] Темиргалиев Н. Т. О вложении некоторых классов функций в $C\left([0,2 \pi]^{m}\right) / /$ Изв. вузов. Матем. 1978. № 8. С. 88-91.

[16] Коляда В.И. Теоремы вложения и неравенства разных метрик для наилучших приближений // Матем. сб. 1977. Т. 102. № 2. С. 195-215.

Бакинский государственный университет, Азербайджан

Поступило

E-mail: nilyasov@yahoo.com

24.11.2000

Исправленный вариант

08.04 .2002 